\title{
The Role of Invariant Functions in Understanding Masses and Mixings
}

\author{
C. Jarlskog \\ Division of Mathematical Physics \\ LTH, Lund University \\ Box 118, S-22100 Lund, Sweden
}

\begin{abstract}
One of the central questions in theoretical particle physics, since already several decades, has been that of "masses and mixings of the quarks. With the entry of neutrino oscillations into the field, the issue of lepton masses has added a new dimension to the problem. In the literature one finds many models of quark and lepton mass matrices. However, the mass and mixing problems remain unsolved.

In this talk I will present my own contributions to this field, however as you may expect without offering a solution. I encourage you to go ahead and think about the problem but be aware of the pitfalls.
\end{abstract}

\section{Introduction}

Understanding the pattern of masses and mixings of the fundamental fermions is generally considered to be extremely important. In the literature one finds several methods of approaching this problem. Let me consider first the quark sector and mention only three approaches.

The first one could be called the "brute force method". One makes ad hoc assumptions about the quark mass matrices, diagonalises them and determines the masses and mixings. An ambitious approach within this category is to "scan" all possible mass matrices. Actually, assuming that there are three families, we know the quark mass matrices from experiments (though not accurately) since about twenty years already! Why then scan and look for something that you already have at hand? The reason, I believe, is that one hopes to find an "elegant" solution which may lead to a deeper insight and understanding.

A second method is to go beyond the standard model. After all, within the Standard Model, the masses and mixings are arbitrary parameters to be determined by experiments. But, for example, in grand unified models one may make predictions for ratios of masses. However, the situation is not yet satisfactory.

A third method is to try to detect numerical relations among either masses or masses and mixings or the mixing angles themselves, hoping that these may indicate a profitable direction for further work. One recent example is the so-called quark-lepton complementarity relations which have recently enjoyed some popularity.

\section{Why invariant functions}

In mid 1980's a question which was being asked was how does one define maximal CP violation in the Standard Model and is CP maximally violated as is parity? As is often the case, there were 
conflicting opinions on what was meant by maximal CP violation. Some authors were advocating that $\mathrm{CP}$ is maximally violated if the $\mathrm{CP}$ phase in the quark mixing matrix is 90 degrees. However, such a definition did not make sense because there is no such unique CP phase. This phase is convention dependent, your CP phase is in general a function of my CP phase and mixing angles. Therefore it was important to understand the structure of CP violation in the Standard Model. It turned out that there is indeed a convention independent invariant for CP violation [1] and this gave rise to the concept of invariant functions of mass matrices [2]. These were further studied in [3].

\section{Definition of invariant functions}

The gauge structure of the Standard Model is defined by the product of three local unitary groups $S U(3) \times S U(2) \times U(1)$. However, in the quark sector there are additional global symmetries, as follows. For $n$ families, we have as usual $n$ left-handed doublets as well as $2 n$ right-handed singlets,

$$
\left(\begin{array}{c}
u_{j} \\
d_{j}
\end{array}\right), \quad u_{j}^{\prime}, \quad d_{j}^{\prime}
$$

where $j=1-n$. Here the $u_{j}$ and $d_{j}$ stand for the left-handed up-type and down-type quarks respectively while their primed counterparts represent the right-handed singlets. We can thus form four vectors

$$
Q_{u}=\left(\begin{array}{c}
u_{1} \\
u_{2} \\
\cdot \\
u_{n}
\end{array}\right), \quad Q_{d}=\left(\begin{array}{c}
d_{1} \\
d_{2} \\
\cdot \\
d_{n}
\end{array}\right), \quad Q_{u}^{\prime}=\left(\begin{array}{c}
u_{1}^{\prime} \\
u_{2}^{\prime} \\
\cdot \\
u_{n}^{\prime}
\end{array}\right), \quad Q_{d}^{\prime}=\left(\begin{array}{c}
d_{1}^{\prime} \\
d_{2}^{\prime} \\
\cdot \\
d_{n}^{\prime}
\end{array}\right)
$$

In special relativity we learn that physics does not depend on the choice of the inertial frame of reference. Something similar happens here where the frames are related to each other by unitary rotations. The transformations between these frames correspond to:

I. Rotating the right-handed up-type quarks by an arbitrary global unitary matrix, i.e., $Q_{u}^{\prime} \rightarrow$ $X_{u}^{\prime} Q_{u}^{\prime}$.

II. Rotating the right-handed down-type quarks by an arbitrary global unitary matrix, $Q_{d}^{\prime} \rightarrow$ $X_{d}^{\prime} Q_{d}^{\prime}$.

III. Rotating the left-handed up-type and down-type quarks simultaneously with the same unitary matrix $Q_{u} \rightarrow X Q_{u}, \quad Q_{d} \rightarrow X Q_{d}$.

The $X$ 's above are all arbitrary unitary matrices.

IV. In addition to the above symmetries there is also the freedom of choosing the phases of the physical quarks.

In this article, due to lack of allocated space, I will only briefly list a number of results that I have obtained, some in collaboration with colleagues, by making use of the above symmetries. 
The first two symmetries can be used to make the quark mass matrices Hermitian without loss of generality, if one so wishes. This is useful for certain applications [4.

The symmetry denoted by III gives the largest class of invariant functions. These functions appear often in physics. Indeed what enters in the Standard Model is not the mass matrices $M_{u}$ and $M_{d}$ for the up-type and down-type quarks respectively but

$$
S_{u} \equiv M_{u} M_{u}^{\dagger}, S_{d} \equiv M_{d} M_{d}^{\dagger}
$$

These are frame dependent. Under III they change

$$
S_{u} \rightarrow X S_{u} X^{\dagger}, \quad S_{d} \rightarrow X S_{d} X^{\dagger}
$$

For the case of three families one may construct an invariant function that automatically keeps track of the 14 conditions that are required to get $\mathrm{CP}$ violation. This function is given by (1], [2]).

$$
\operatorname{det}\left[S_{u}, S_{d}\right]=2 i J v\left(S_{u}\right) v\left(S_{d}\right)
$$

where $\mathrm{J}$ is an invariant whose magnitude equals twice the area of any of the six by now well-known unitarity triangles [5]. The quantities $v\left(S_{u}\right)$ and $v\left(S_{d}\right)$ are given by $v\left(S_{u}\right)=\left(m_{u}^{2}-m_{c}^{2}\right)\left(m_{c}^{2}-\right.$ $\left.m_{t}^{2}\right)\left(m_{t}^{2}-m_{u}^{2}\right)$ and $v\left(S_{d}\right)=\left(m_{d}^{2}-m_{s}^{2}\right)\left(m_{s}^{2}-m_{b}^{2}\right)\left(m_{b}^{2}-m_{d}^{2}\right)$ (for a pedagogical discussion see [6] $)$.

Looking into literature, we see the determinant in Eq.(5-5) in essentially every computation involving $\mathrm{CP}$ violation, in all its glory when all the six quarks enter on equal footing but otherwise in a well-defined truncated form, where some factors are missing due to assumptions made in the calculation 3. Examples of the first kind are the renormalization of the $\theta$-parameter of QCD by the electroweak interactions and the calculation of the baryon asymmetry of the universe in the standard model. An example of the second kind is the computation of the electric dipole moment of a quark, say the down quark. Since in such a computation, the down quark appears in the external legs, it is tacitly assumed that we know the identity of this quark, i.e., $m_{d} \neq m_{s}$ and $m_{d} \neq m_{b}$. Therefore the factors $\left(m_{d}^{2}-m_{s}^{2}\right)$ and $\left(m_{b}^{2}-m_{d}^{2}\right)$ are missing but all the other factors are present.

The above commutator is the simplest in a family of commutators of functions of mass matrices [2] which I will not discuss here. These give us new invariant functions, some of which enter in the computation of neutrino oscillations [7]. In addition, for example, the absolute values of the elements of the quark mixing matrix are measurable quantities and thus invariant functions. These functions were constructed in [3] (see also [8]).

\section{Textures and quark-lepton relations}

Theorists love zeros. Generally zeros in the mass matrices make their diagonalisation and thus life simpler. However, we have seen that such zeros are not invariants! If you give me mass matrices with some zeros I may use symmetry III whereby the zeros could evaporate. In the case of leptons in most models one ends up with an effective three-by-three mass matrix for the neutrinos. In this case there is no known justification for making assumptions about how the neutrino mass matrix should look like in a frame where the charged-lepton mass matrix is diagonal. This frame is highly 
"fine-tuned". You have to ask yourself how does the Standard Model find this very special frame in an infinite class of all possible frames?

My problem with the quark-lepton complementarity relations (see the talk by Mohapatra where the subject is briefly discussed) is again that such relations are not invariants. Mixing angles, for three or more families, are convention dependent. Your mixing angles and phases are in general functions of my corresponding quantities. Even the order in which the Euler-type rotations are introduced is not preset. Let us take a very simple example. As mentioned before, in most models the leptonic mixing matrix appears essentially in the same form as the quark mixing matrix. In a simplified notation we have

$$
(e, \mu, \tau) U\left(\begin{array}{l}
\nu_{1} \\
\nu_{2} \\
\nu_{3}
\end{array}\right)
$$

Suppose that we decide to write the mixing matrix $U$ in two different but equivalent forms

$$
V=R_{23} R_{13} R_{12}, \quad V^{\prime}=R_{12}^{\prime} R_{13}^{\prime} R_{23}^{\prime}
$$

where $R_{j k}$ denotes a rotation in the $j k$-plane by the angle $\theta_{j k}$ while its primed counterpart denotes the same rotation by the angle $\theta_{j k}^{\prime}$. In this example the second matrix is obtained from the first one by transposition and redefinition of the angles, $V^{\prime}=\tilde{V}\left(\theta_{j k} \rightarrow-\theta_{j k}^{\prime}\right)$.

Now compare the two limits $\theta_{13} \rightarrow 0$ and $\theta_{13}^{\prime}$. For nondegenerate neutrinos they are physically inequivalent. In one case the electron couples only to two physical neutrinos while the muon and the tau couple to all three. In the other case it is the tau that couples only to two neutrinos. This simple example shows that it doesn't make sense to compare mixing angles as these are ill-defined. The problems become more serious when one adds mixing angles from the quark and lepton sectors. We don't even know whether we should add or subtract, what are the relative phases, etc. Some of these issues have been discussed in [9].

\section{Outlook}

The issue of masses and mixings remains an unsolved problem that deserves our attention. However, we should always keep in mind that important results can't be frame dependent. So, if you have an important message to transmit, you should be able to formulate it in an invariant form. Unfortunately, I have no space to describe some work done along this line [10], nor some work that I have done on parameterisation of unitary matrices [11] which may be of interest to you.

\section{Acknowledgements}

I wish to thank the Organisers of the SNOW 2006, specially Tommy Ohlsson, for a wonderful meeting where this talk was presented. 


\section{References}

[1] C. Jarlskog, Phys. Rev. Lett. $\underline{55}$ (1985) 1039

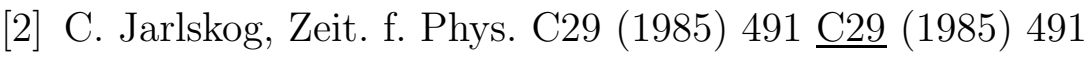

[3] C. Jarlskog, Phys. Rev. D35 (1987) 1685; ibid D36 (1987) 2128

[4] P. H. Frampton and C. Jarlskog, Phys. Lett. B 154 (1985) 421

[5] C. Jarlskog and R. Stora, Phys. Lett. B208 (1988) 268

[6] C. Jarlskog in CP Violation, Ed. C. Jarlskog (World Scientific, 1989) p. 3

[7] C. Jarlskog, Phys. Lett. B 609 (2005) 323

[8] C Jarlskog and A Kleppe, Nucl. Phys. B286 (1987) 245

[9] C. Jarlskog, Phys. Lett. B 625 (2005) 63

[10] P. Harrison and W. G. Scott, , Phys. Lett. B 628 (2005) 93

[11] C. Jarlskog, J. Math. Phys. 46 (2005) 103508;

ibid 47 (2006) 013507 